Received: 20.04 .2021

Revised: 17.06 .2021

Accepted: 25.06 .2021

DOI: $10.17804 / 2410-9908.2021 .3 .006-016$

\title{
PRESSURE DEPENDENCE OF SHEAR STRESS DURING HIGH-PRESSURE TORSION OF THE Au-Co ALLOYS IN LIQUID NITROGEN
}

\author{
T. P. Tolmachev ${ }^{1,2, \text { a)*}^{*}, \text { V. P. Pilyugin }}{ }^{2,3, \mathrm{~b})}$, A. M. Patselov ${ }^{2, \mathrm{c})}$, \\ N. V. Nikolayeva ${ }^{2}$, A. M. Vlasova ${ }^{2, d)}$ \\ ${ }^{1}$ Institute of Engineering Science, Ural Branch of the Russian Academy of Sciences, 620049, \\ 34 Komsomolskaya st., Ekaterinburg, Russian Federation \\ ${ }^{2}$ M.N. Miheev Institute of Metal Physics, Ural Branch of the Russian Academy of Sciences, \\ 620108, 18 S. Kovalevskoy St., Ekaterinburg, Russian Federation \\ ${ }^{3}$ Ural Federal University, 620002, 19 Mira street, Ekaterinburg, Russian Federation

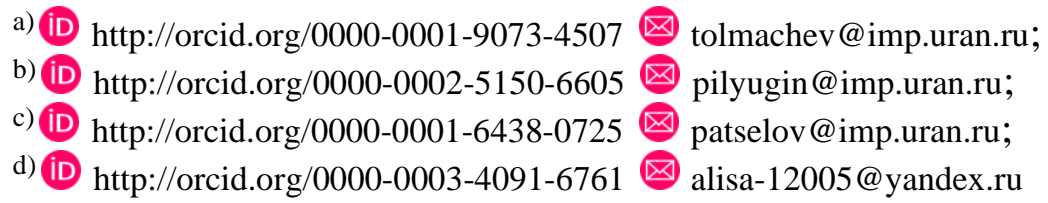 \\ *Corresponding author. E-mail: tolmachev@imp.uran.ru \\ Address for correspondence: ul. S. Kovalevskoy, 18, Ekaterinburg, 620108, Russian Federation \\ Tel.: +7 (343) 37838 05; fax: +7 (343) 3745244 .
}

Measurements of the shear stress were carried out during mechanical alloying by highpressure torsion of gold-cobalt powder mixing under cryogenic temperature at various pressures for the first time. The pressures were about 5, 8, 10 and $12 \mathrm{GPa}$. The $\mathrm{Au}-\mathrm{Co}$ system is characterized by restricted solubility-and difference in properties of the constituents. It was found that, in comparison with the data obtained at room torsion temperature, the values of the shear stress are higher both at the stage of the initial intensive growth and subsequently, upon reaching saturation. With an increase in pressure of low-temperature mechanical alloying, a corresponding increase in the resistance to shear strain is observed at all stages of processing. In this case, the shape of the curves at each new pressure changes, as does the staging of the strain dependence. This reflects the extreme heterogeneity of the structure formation and phase composition processes of the non-equilibrium alloy. The most intense reflections in the X-ray diffractogram correspond to supersaturated fcc solid solution of cobalt based on gold-base matrix. Also as a result of mechanical alloying, the components of the system are evenly distributed on the fracture surface of the alloy.

Keywords: mechanical alloying, high-pressure torsion, cryogenic deformation, restricted solubility $\mathrm{Au}-\mathrm{Co}$ system, in situ shear stress vs. strain.

\section{Acknowledgments}

The research was financially supported by the RFBR under project No. 19-32-60039 and partially under the state assignment from the Ministry of Education and Science of the Russian Federation (theme Pressure, No. AAAA-A18-118020190104-3). The materials were produced and processed at the IMP UB RAS, Ekaterinburg. The electron microscope investigations were made with the use of the equipment of the Testing Center of Nanotechnology and Advanced Materials shared access center at the IMP UB RAS, Ekaterinburg. 


\section{References}

1. Okamoto H., Massalski T.B., Hasebe M., Nishizawa T. The Au-Co (Gold-Cobalt) system. Bulletin of Alloy Phase Diagrams, 1985, vol. 6, pp. 449-454. DOI: 10.1007/BF02869509.

2. $\quad$ Barabash O.M., Koval Yu.N. Struktura i svoystva metallov $i$ splavov [Structure and Properties of Metals and Alloys]. Kiev, Naukova Dumka Publ., 1986, 599 p. (In Russian).

3. Miedema A.R., De Chatel P.F., De Boer F.R. Cohesion in alloys - fundamentals of a semiempirical model. Physica B, 1980, vol. 100, pp. 1-28. DOI: 10.1016/0378-4363(80)90054-6.

4. M. E. Drits, ed. Svoystva elementov [Properties of Elements: A Handbook]. Moscow, Metallurgiya Publ., 1985. (In Russian).

5. Yin J., Chen G., Tang H., Qu X. Athermal $\omega$ phase transformation from $\beta$ in Ti-28Ta driven by ultra-rapid quenching. International Journal of Refractory Metals and Hard Materials, 2020, vol. 92, pp. 105250. DOI: 10.1016/j.ijrmhm.2020.105250.

6. Meng X., Zhang D., Zhang W., Qiu C., Liang G., Chen J. Microstructure and mechanical properties of a high-Zn aluminum alloy prepared by melt spinning and extrusion. Journal of Alloys and Compounds, 2020, vol. 819, pp. 152990. DOI: 10.1016/j.jallcom.2019.152990.

7. Belkacemi L.T., Meslin E., Décamps B., Crocombette J.-P., Tissot O., Vandenberghe T., Desgardin P., Sauvage T., Berthier C. Role of displacement cascades in Ni clustering in a ferritic Fe-3.3 at\% Ni model alloy: Comparison of heavy and light particle irradiations. Scripta Materialia, 2020, vol. 188, pp. 169-173. DOI: 10.1016/j.scriptamat.2020.07.031.

8. Tejeda-Ochoa A., Kametani N., Carreño-Gallardo C., Ledezma-Sillas J.E., Adachi N., Todaka Y., Herrera-Ramirez J.M. Formation of a metastable fcc phase and high $\mathrm{Mg}$ solubility in the Ti-Mg system by mechanical alloying. Powder Technology, 2020, vol. 374, pp. 348-352. DOI: $10.1016 /$ j.powtec.2020.07.053.

9. Dobromyslov A.V., Taluts N.I., Pilyugin V.P. Severe plastic deformation by high-pressure torsion of Hf and Hf-Ti alloys. International Journal of Refractory Metals and Hard Materials, 2020, vol. 93, pp. 105354. DOI: 10.1016/j.ijrmhm.2020.105354.

10. Sun J., Ke Q., Chen W. Material instability under localized severe plastic deformation during high speed turning of titanium alloy Ti-6.5AL-2Zr-1Mo-1V. Journal of Materials Processing Technology, 2019, vol. 264, pp. 119-128. DOI: 10.1016/j.jmatprotec.2018.09.002.

11. Bridgman P.W. Effects of High Shearing Stress Combined with High Hydrostatic Pressure. Physical Review, 1935, vol. 48, pp. 825-847. DOI: 10.1103/PhysRev.48.825.

12. Zhilyaev A.P., Langdon T.G. Using high-pressure torsion for metal processing: Fundamentals and applications. Progress in Materials Science, 2008, vol. 53, pp. 893-979. DOI: $10.1016 /$ j.pmatsci.2008.03.002.

13. Glezer A., Kozlov E., Koneva N., Popova N., Kurzina I. Plastic Deformation of Nanostructured Materials, Boca Raton, CRC Press, 2017. DOI: 10.1201/9781315111964.

14. Teplov V.A., Pilugin V.P., Gaviko V.S., Chernyshov E.G. Non-equilibrium solid solution and nanocrystal structure of $\mathrm{Fe}-\mathrm{Cu}$ alloy after plastic deformation under pressure. Philosophical Magazine B, 1993, vol. 68, pp. 877-881, DOI: 10.1080/13642819308217944.

15. Nomura R., Uesugi K. Note: High-pressure in situ X-ray laminography using diamond anvil cell. Review of Scientific Instruments, 2016, vol. 87, pp. 046105. DOI: 1063/1.4948315.

16. Towle L.C., Riecker R.E. Shear Strength of Grossly Deformed Solids. Science, 1969, vol. 163, pp. 41-47. DOI: 10.1126/science.163.3862.41.

17. Sundeev R.V., Shalimova A.V., Glezer A.M., Pechina E.A., Gorshenkov M.V., Nosova G.I. In situ observation of the "crystalline $\Rightarrow$ amorphous state" phase transformation in $\mathrm{Ti} 2 \mathrm{NiCu}$ upon high-pressure torsion. Materials Science and Engineering: A, 2017, vol. 679. pp. 1-6. DOI: $10.1016 /$ j.msea.2016.10.028.

18. Sundeev R.V., Shalimova A.V., Sitnikov N.N., Chernogorova O.P., Glezer A.M., Presnyakov M.Y., Karateev I.A., Pechina E.A., Shelyakov A.V. Effect of high-pressure torsion on the structure and 
properties of the natural layered amorphous-crystalline Ti2NiCu composite. Journal of Alloys and Compounds, 2020, vol. 845, pp 156273. DOI: 10.1016/j.jallcom.2020.156273.

19. Tolmachev T.P., Pilyugin V.P., Ancharov A.I., Chernyshov E. G., Patselov A. M. The formation, structure, and properties of the $\mathrm{Au}-\mathrm{Co}$ alloys produced by severe plastic deformation under pressure. The Physics of Metals and Metallography, 2016, vol. 117, pp. 135-142. DOI: 10.1134/S0031918X16020125.

20. Tolmachev T.P., Pilyugin V.P., Patselov A.M., Plotnikov A.V., Churbaev R.V. Shear stress in high-pressure torsion and vickers hardness of Au-Co alloys. AIP Conference Proceedings, 2020, vol. 2315, pp. 040046. DOI: 10.1063/5.0036671.

21. Tolmachev T.P., Pilyugin V.P., Patselov A.M. Solov'eva Yu.V., Churbaev R.V., Plotnikov A.V. Structural features of the $\mathrm{Au}-\mathrm{Co}$ alloy after mechanical alloying at cryo- and room temperatures according to X-ray diffractometry. Russian Physics Journal, 2021. (In press). 
Подана в журнал: 20.04.2021

УДК 669.21'25:539.89

DOI: $10.17804 / 2410-9908.2021 .3 .006-016$

\title{
ВЛИЯНИЕ БАРИЧЕСКИХ УСЛОВИЙ НА ЗАВИСИМОСТЬ НАПРЯЖЕНИЯ СДВИГА ПРИ КРУЧЕНИИ ПОД ВЫСОКИМ ДАВЛЕНИЕМ В ЖИДКОМ АЗОТЕ СПЛАВОВ СИСТЕМЫ Аu-Со
}

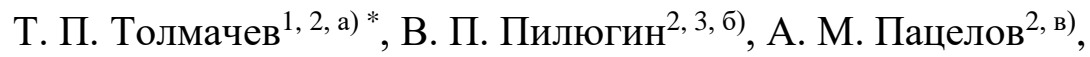 \\ Н. В. Николаева ${ }^{2}$, А. М. Власова ${ }^{2, \text { г) }}$ \\ ${ }^{1}$ Институт машиноведения УрО РАН, Екатеринбург, Российская Федеращия \\ ${ }^{2}$ Институт физики металлов УрО РАН, Екатеринбург, Российская Федерация \\ ${ }^{3}$ Уральский федеральный университет, Екатеринбург, Российская Федерачиия \\ a) iD http://orcid.org/0000-0001-9073-4507 tolmachev@imp.uran.ru; \\ б) (iD http://orcid.org/0000-0002-5150-6605 pilyugin@imp.uran.ru; \\ в) (iD http://orcid.org/0000-0001-6438-0725 patselov@imp.uran.ru; \\ г) iD http://orcid.org/0000-0003-4091-6761 @ alisa-12005@yandex.ru

\begin{abstract}
*Ответственный автор. Электронная почта: tolmachev@imp.uran.ru Адрес для переписки: ул. С. Ковалевской, 18, Екатеринбург, 620108, Российская Федерация
\end{abstract} \\ Тел.: +7 (343) 378-35-05; факс: (343) 374-52-44
}

Впервые проведено измерение напряжения сдвига при механическом сплавлении кручением под высоким давлением в условиях криогенной температуры компонентов системы с ограниченной растворимостью золото-кобальт при различных значениях давления обработки, которые составляли около 5, 8, 10 и 12 ГПа. Установлено, что по сравнению с данными, полученными при кручении в условиях комнатной температуры, значения напряжения сдвига выше как на стадии начального интенсивного роста, так и впоследствии - при выходе на насыщение. С повышением давления при низкотемпературном кручении наблюдается соответствующее возрастание сопротивления деформации сдвига на всех этапах обработки. При этом вид кривых при каждом новом давлении изменяется, как изменяется и стадийность деформационной зависимости, что отражает крайнюю неоднородность процессов формирования структуры и фазового состава получаемого сплава. По рентгеновским данным установлено, что в результате низкотемпературной совместной деформации компонентов изучаемой системы формируется преимущественно пересыщенный ГЦК-твердый раствор кобальта на основе золота. Компоненты системы в результате механического сплавления распределены равномерно на поверхности излома сплава.

Ключевые слова: механическое сплавление, кручение под высоким давлением, криодеформация, система ограниченной растворимости золото-кобальт, in situ напряжение сдвига от величины деформации.

\section{1. Введение}

Система Au-Со характеризуется ограниченной растворимостью, практически нулевой при комнатной и более низких температурах $[1,2]$. Даже в жидком состоянии для такой системы уже характерно расслоение вместо однородного твердого раствора согласно модельным представлениям [3]. Кроме того, кобальт и золото различаются по своим физикомеханическим характеристикам [2, 4]. Системы ограниченной растворимости, в которых один из компонентов - благородный металл, а второй - ферромагнитный, представляют собой интерес в качестве материалов с управляемой структурой и свойствами. Формируя метастабильные фазы в таких системах, можно последующей обработкой достигать состояний 
с заданными параметрами структуры, фазового состава и физико-механическими свойствами. К методам формирования метастабильных фаз относятся следующие: сверхбыстрая закалка из расплава [5,6], облучение высокоэнергетическими частицами [7], механическое сплавление [8], мегапластическая (большая или интенсивная пластическая) деформация $[9,10]$. Механическое сплавление представляет собой обработку порошковой смеси сплавляемых компонентов с помощью большой пластической деформации, в результате чего формируются твердые растворы и/или соединения. В качестве сплавляемых компонентов часто используют металлы и химические соединения. Наиболее распространенный метод механического сплавления - помол в шаровой мельнице.

Метод кручения под высоким квазигидростатическим давлением в камере Бриджмена $[11,12]$, изначально относившийся к методам мегапластической деформации $[12,13]$, позволяет осуществлять и механическое сплавление [14]. При этом готовые сплавы представляют собой образцы в цельном объемном виде, пригодном для дальнейшего исследования структуры и физико-механических свойств без дополнительного воздействия. Данный метод позволяет осуществлять и варьирование параметров обработки, что позволяет оказывать воздействие на характеристики структуры и свойств еще на стадии получения готового образца. Большинство исследований свойств механически синтезированных сплавов относятся к периоду после формирования сплава, однако существуют методы измерения и непосредственно в процессе обработки, например $[15,16]$. К таковым относят измерение напряжения сдвига во время деформации [16-18].

Влияние температурного режима большой пластической деформации состоит в изменении преимущественных механизмов формирования деформационно-индуцированной структуры, а в случае механического сплавления - в деформационно-индуцированном формировании новых фаз. Ранее было установлено, что в системе золото-кобальт снижение температуры кручения до криогенной, соответствующей температуре кипения жидкого азота, приводит к формированию сплавов с большей долей кобальта в пересыщенном ГЦКтвердом растворе на основе золота, а также к повышению механических свойств итогового сплава [19].

Влияние изменения давления на зависимость напряжения сдвига во время деформирования рассматриваемой системы ранее исследовалось в условиях комнатной температуры [20]. В целом, было установлено увеличение значений напряжения сдвига при выходе деформационной зависимости на стационарную стадию с ростом числа оборотов наковальни. Качественно же вид кривых не изменялся с ростом давления.

Цель работы - выявление влияния изменения барических условий кручения под высоким давлением при криогенной температуре компонентов системы ограниченной растворимости $\mathrm{Au-Co} \mathrm{на} \mathrm{напряжение} \mathrm{сдвига} \mathrm{во} \mathrm{время} \mathrm{деформации.}$

\section{2. Методы и материалы}

Кобальт чистотой 99,60 \% и золото чистотой 99,99 \% в исходном состоянии представляли собой порошки с размером частиц около 50 мкм и около 300 мкм соответственно. Порошковые компоненты смешивали в эквиатомной пропорции и затем подвергали деформации кручения (сдвига) под высоким давлением (КВД) на вращаемых наковальнях Бриджмена при температуре кипящего жидкого азота (около 77,4 К). Тип наковален - с плоскими поверхностями рабочих площадок диаметром 5 мм; материал наковален - ВК-6 твердостью 92 HRC.

Мегапластическую деформацию порошковых смесей проводили при различных давлениях: 5-6, 8, 10 и 12 ГПа. Образцы сплавов после механосплавления имели дискообразную форму диаметром 5 мм и толщиной около 0,1 мм. In situ под давлением измеряли сопротивление деформации сдвига образцов от угла поворота с использованием электронного динамометра АЦДС-1И-1 следующим образом. Во время обработки нижняя наковальня приводи- 
лась во вращение, в то время как верхняя с помощью рычага передавала на датчик усилие. Напряжение сдвига $\tau$ вычисляли по формуле [16]:

$$
\tau=\frac{3 L}{2 \pi a^{3}} F
$$

$\tau=\frac{3 \mathrm{~L}}{2 \pi \mathrm{a}^{3}} \cdot$ Fгде $\tau$ - напряжение сдвига, ГПа; $a$ - радиус наковальни, м; $L-$ длина рычага от наковальни до динамометра, м; $F$ - усилие на динамометре, кН.

Рентгеновский дифракционный анализ проводили на образце сплава после 30 оборотов наковальни Бриджмена в жидком азоте при давлении 8 ГПа. Съемку проводили в излучении кобальта, в геометрии на отражение со всей поверхности образца на аппарате ДРОН-3. Элементный анализ проводили с помощью сканирующей электронной микроскопии на аппарате QUANTA-200 с приставкой для энерго-дисперсионного анализа EDAX.

\section{3. Результаты и обсуждение}

По данным рентгеновского дифракционного анализа, в результате низкотемпературной деформации под давлением 8 ГПа сформировался сплав, содержащий фазы твердых растворов золота и кобальта (рис. 1). Помимо кристаллических фаз имеется и рентгеноаморфная [21]. По наиболее интенсивным рефлексам можно идентифицировать, что преимущественная по удельному объему кристаллическая составляющая фазового состава сплава это ГЦК-твердый раствор.

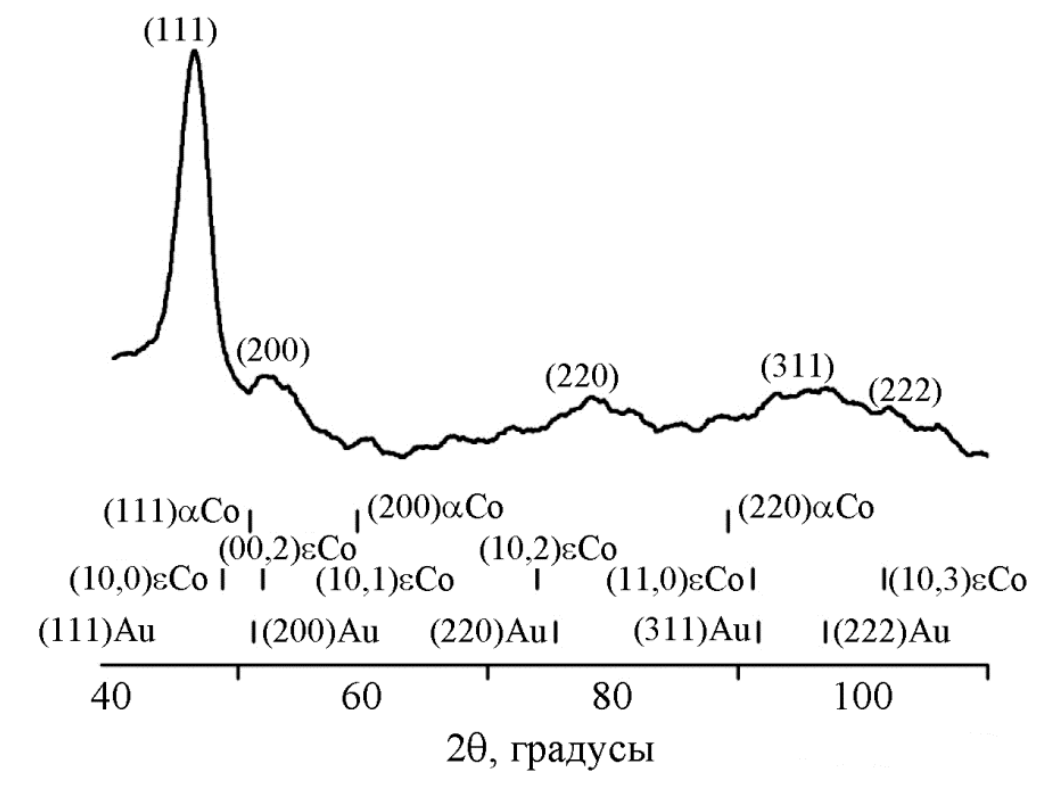

Рис. 1. Рентгеновская дифрактограмма сплава золото-кобальт в результате низкотемпературного кручения под давлением 8 ГПа

Установлено, что фаза твердого раствора имеет период кристаллической решетки 0,397 нм, что соответствует, по разным оценкам, 20-28 ат. \% [21]. Близкое значение находится на границе равновесной растворимости в данной системе [2], но только при температурах около $1000{ }^{\circ} \mathrm{C}$. Настоящий же сплав был получен при температуре кипения жидкого азота, т.е. около $77,4 \mathrm{~K}$.

Построение карт распределения компонентов изучаемой системы на изображении излома сплава (рис. 2 a), полученного крио-КВД при 6 ГПа и 30 оборотов наковальни, показало, распределение золота (рис. 2 б) и кобальта практически однородное (рис. 2 в). 


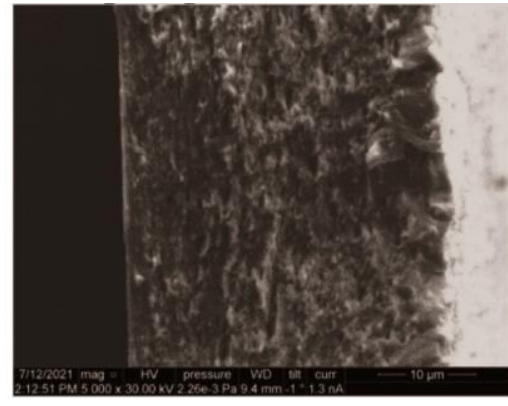

$a$

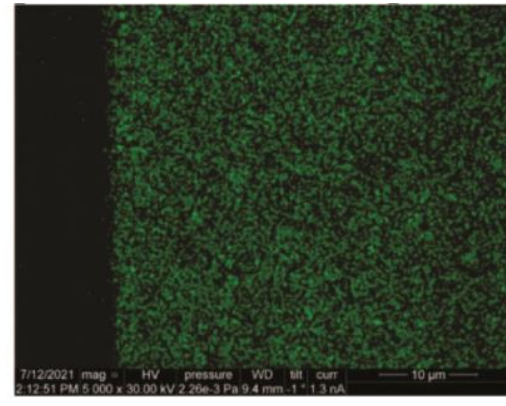

6

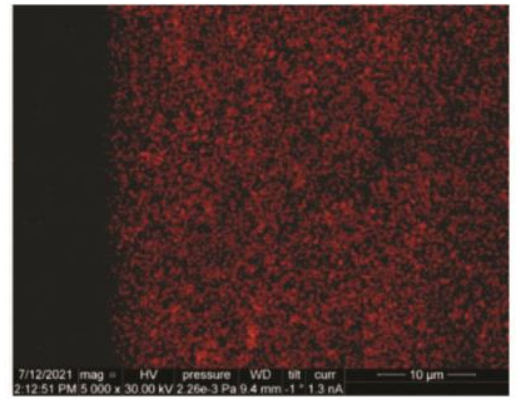

8

Рис. 2. Карты распределения компонентов системы золото-кобальт на диаметральном срезе после механического сплавления при температуре кипящего жидкого азота и давлении 6 ГПа: $a$ - изображение среза; $\sigma$ - распределение золота; 6 - распределение кобальта

Таким образом, в результате криодеформации на 30 оборотов смеси порошков Аu и Со растворяемые компоненты равномерно распределяются по объему образца, при этом в составе сплава формируются фазы, наиболее интенсивная из которых, по данным рентгеновской дифрактометрии, представляет собой пересыщенный ГЦК-твердый раствор замещения кобальта в матрице на основе золота.

Непосредственно процесс формирования сплава при КВД, хотя бы частично, можно проследить по его сопротивлению деформации сдвига. Зависимости напряжения сдвига при совместной деформации показывают вклад добавления растворяемого компонента и влияние температуры деформирования смеси на деформационную кривую.

При КВД золота без добавления кобальта напряжение сдвига резко возрастает сразу же после начала обработки и достигает значений около 2,8 ГПа (рис. 3). Далее, уже после первого оборота наковальни обрабатываемый материал перестает показывать рост сопротивления сдвиговой деформации. Данную зависимость снимали при давлении 8 ГПа.

В случае же механосплавления смеси золота и кобальта при 6 ГПа и комнатной температуре [20] из шихты с эквиатомным соотношением компонентов вместо интенсивного возрастания момента кручения наблюдается его более плавный рост (рис. 3). При значении около 2,8 ГПа рост напряжения сдвига не заканчивается, а продолжается еще достаточно большой промежуток времени. За это время наковальни успевают осуществить 8-10 полных оборотов. Только после достижения 10 оборотов достигается состояние насыщения.

Низкотемпературная зависимость напряжения сдвига смеси компонентов при 6 ГПа свидетельствует, что качественно вид кривой не изменился, а выход на насыщение происходит около 4,2 ГПа (рис. 3 и 4). Однако после 22 оборотов напряжение сдвига выходит из состояния динамического равновесия и продолжает возрастать (рис. 3 и 4).

Повышение давления обработки до 8 ГПа приводит к тому, что зависимость напряжения сдвига изменяется, в том числе и на качественном уровне (рис. 4). После начального интенсивного роста и дальнейшего замедления роста $\tau$ выхода на стационарную стадию не наблюдается. Вместо последнего происходит небольшое снижение, и только после него, при обработке в 10-12 оборотов, происходит выход на динамическое равновесие при значениях напряжения сдвига около 7,2 ГПа.

Механическое сплавление при 10 ГПа не выявляет каких-либо качественных отличий для напряжения сдвига от стандартного для обработки при комнатной температуре вида кривой (рис. 3) [19, 20]: стадия начального интенсивного роста напряжения сдвига сменяется его замедлением с последующим выходом на динамическое равновесие, которое происходит после 5-6 оборотов и сохраняется вблизи значений около 9 ГПа на всем протяжении дальнейшей деформации (рис. 4). 


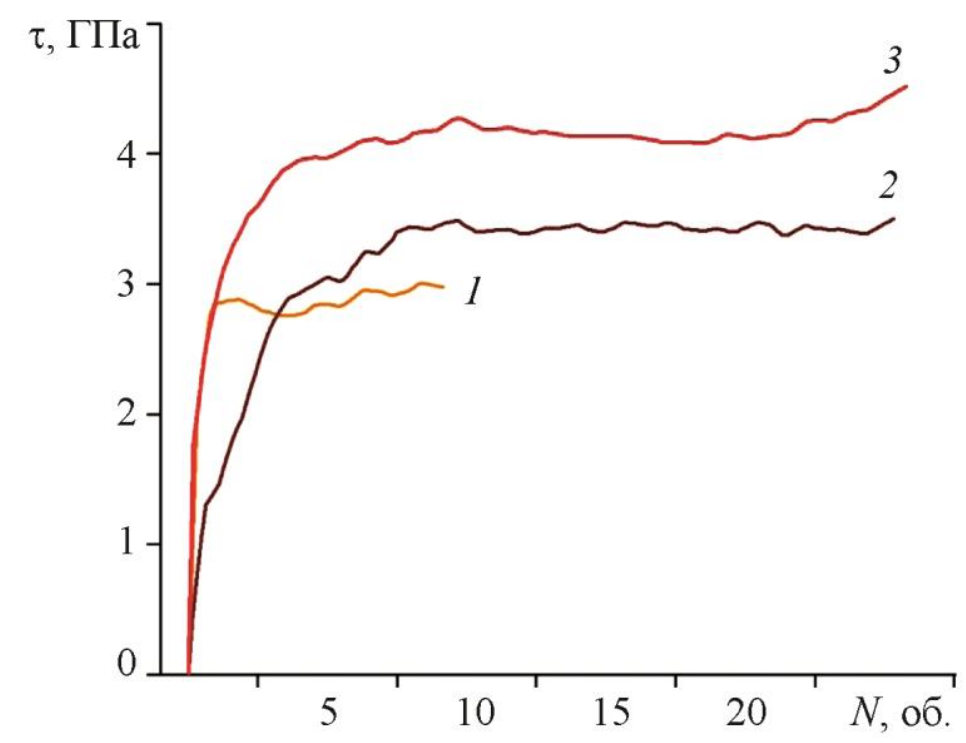

Рис. 3. Зависимости напряжения сдвига при кручении под высоким давлением от числа оборотов наковальни Бриджмена: 1 - золото без добавления кобальта в условиях комнатной температуры и давлении 8 ГПа; 2 - смесь Аu-Со при комнатной температуре и давлении 6 ГПа; 3 - смесь Аu-Со при температуре жидкого азота и давлении 6 ГПа

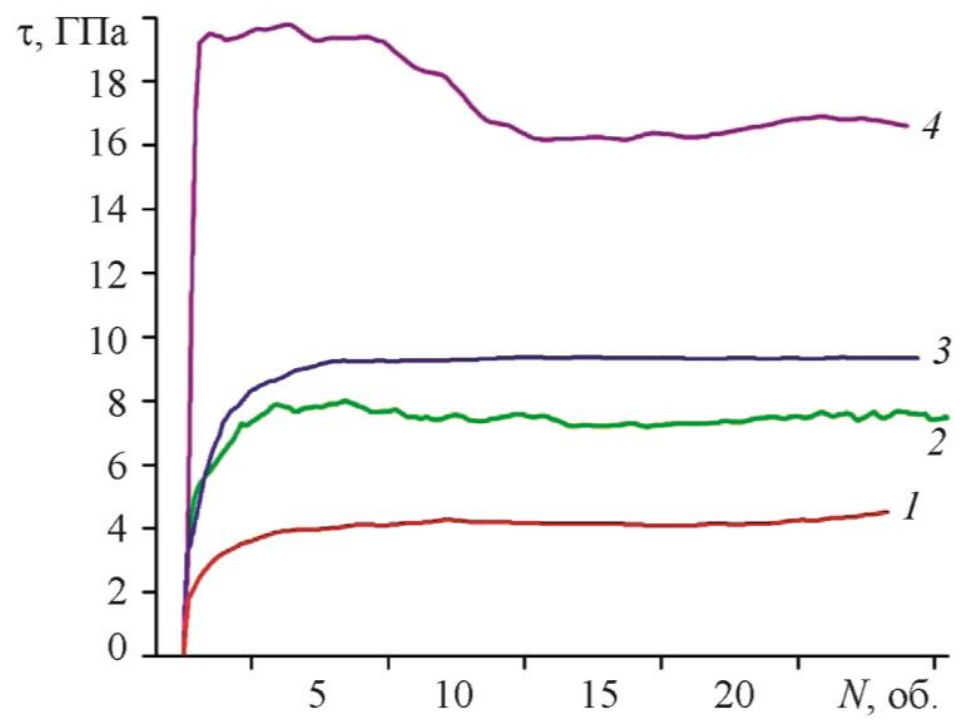

Рис. 4. Зависимости напряжения сдвига от числа оборотов наковальни Бриджмена во время деформации кручением под высоким давлением при температуре жидкого азота и при давлении: 1 - 5 ГПа; 2 - 8 ГПа; 3 - 10 ГПа; 4 - 12 ГПа

КВД при 12 ГПа привело к еще более значительному изменению вида кривой напряжения сдвига (рис. 4). Подобно зависимости при 8 ГПа, после интенсивного роста и его замедления, происходит снижение, причем более значительное по величине. Выход на динамическое равновесие наблюдается при 12 оборотах наковальни и слабо изменяется вблизи значений 16,5 ГПа на протяжении дальнейшей деформации. Отметим что, по данным [19], повышение давления до 12 ГПа приводило к увеличению доли кобальта в составе твердого раствора $\mathrm{Au}-\mathrm{Co}$.

Таким образом, напряжение сдвига при КВД, измеренное в разных температурных условиях, начинает различаться уже при первом обороте наковальни: в жидком азоте дефор- 
мационная кривая возрастает интенсивнее по сравнению с деформацией при комнатной температуре.

Вместе с тем необходимо учитывать, что зависимости напряжения сдвига от деформации отражают интегральные процессы структурных и фазовых изменений. Особенно это характерно для начальной стадии, когда на периферии образца может быть уже достигнуто ультрамелкозернистое состояние, а в центре образца - еще нет. Отметим, что после достижения большого числа оборотов наковальни структурно-фазовые превращения, характерные для периферии, происходят и в центре образца. Если при комнатной температуре КВД происходит стабилизация сопротивления деформации сдвига на одном уровне, то в условиях криогенных температур практически все деформационные кривые проявляют отличное поведение. Обусловлено это и сменой преимущественных механизмов деформационного измельчения структуры каждого компонента и деформационно-индуцированных процессов взаимного растворения, а также влиянием температурных условий на функционирование оборудования, материалы которого также не исключают деформационных процессов. Последнее может затруднять вычленение и интерпретацию результатов измерений. При этом низкотемпературный режим интенсивной пластической деформации приводит к большему сопротивлению сдвиговой деформации по сравнению с обработкой при комнатной температуре, а также к более сложному виду in situ зависимостей напряжения сдвига.

\section{4. Заключение}

Методом мегапластической деформации кручением под высоким давлением на 30 оборотов при криогенной температуре были получены сплавы Au-Co из эквиатомной смеси компонентов в порошковом виде. Давление обработки варьировалось с шагом в 2 ГПа от 5-6 и до 12 ГПа. Во время КВД снимали зависимость напряжения сдвига от величины деформации. Установлено, что по сравнению с данными, полученными в условиях комнатной температуры мегапластической деформации, значения напряжения сдвига выше как на стадии начального роста, так и при выходе их на насыщение. С повышением давления при низкотемпературном режиме наблюдается соответствующее возрастание сопротивления деформации сдвига на всех этапах обработки, как и при комнатной КВД. При этом вид кривых при каждом новом давлении изменяется, как изменяется и стадийность деформационной зависимости, что отражает крайнюю неоднородность процессов формирования структуры и фазового состава получаемого сплава. Наиболее интенсивным рентгеновским линиям на дифрактограмме соответствует фаза пересыщенного ГЦК-твердого раствора $\mathrm{Au}-\mathrm{Co}$. Компоненты системы в результате деформации распределены равномерно на поверхностях изломов сплавов.

\section{Благодарность}

Исследование выполнено при финансовой поддержке РФФИ в рамках научного проекта № 19-32-60039 и частично в рамках государственного задания МИНОБРНАУКИ России (тема "Давление», № АААА-А18-118020190104-3). Получение и обработка материалов производились на базе ИФМ УрО РАН, г. Екатеринбург. Электронно-микроскопические исследования проводились на оборудовании ЦКП «Испьтательный центр нанотехнологий и перспективных материалов» ИФМ УрО РАН, г. Екатеринбург.

\section{Литература}

1. The Au-Co (Gold-Cobalt) system / H. Okamoto, T. B. Massalski, M. Hasebe, T. Nishizawa // Bulletin of Alloy Phase Diagrams. - 1985. - Vol. 6. - P. 449-454. - DOI: 10.1007/BF02869509.

2. Барабаш О. М., Коваль Ю. Н. Структура и свойства металлов и сплавов. - Киев : Наукова думка, 1986. - 599 с. 
3. Miedema A. R., De Chatel P. F., De Boer F. R. Cohesion in alloys - fundamentals of a semi-empirical model // Physica B. - 1980. - Vol. 100. - P. 1-28. - DOI: 10.1016/03784363(80)90054-6.

4. Свойства элементов : справочник / М. Е. Дриц, П. Б. Будберг, Г. С. Бурханов, А. М. Дриц, В. М. Пановко / под ред. М. Е. Дрица - М. : Металлургия, 1985 - 672 с.

5. Athermal $\omega$ phase transformation from $\beta$ in Ti-28Ta driven by ultra-rapid quenching / J. Yin, G. Chen, H. Tang, X. Qu // International Journal of Refractory Metals and Hard Materials. 2020. - Vol. 92. - P. 105250. - DOI: 10.1016/j.ijrmhm.2020.105250.

6. Microstructure and mechanical properties of a high-Zn aluminum alloy prepared by melt spinning and extrusion / X. Meng, D. Zhang, W. Zhang, C. Qiu, G. Liang, J. Chen // Journal of Alloys and Compounds. - 2020. - Vol. 819. - P. 152990. - 10.1016/j.jallcom.2019.152990.

7. Role of displacement cascades in $\mathrm{Ni}$ clustering in a ferritic $\mathrm{Fe}-3.3$ at\% $\mathrm{Ni}$ model alloy: Comparison of heavy and light particle irradiations / L. T. Belkacemi, E. Meslin, B. Décamps, J.-P. Crocombette, O. Tissot, T. Vandenberghe, P. Desgardin, T. Sauvage, C. Berthier // Scripta Materialia. - 2020. - Vol. 188. - P. 169-173. - DOI: 10.1016/j.scriptamat.2020.07.031.

8. Formation of a metastable fcc phase and high $\mathrm{Mg}$ solubility in the Ti-Mg system by mechanical alloying / A. Tejeda-Ochoa, N. Kametani, C. Carreño-Gallardo, J. E. Ledezma-Sillas, N. Adachi, Y. Todaka, J. M. Herrera-Ramirez // Powder Technology. - 2020. - Vol. 374. - P. 348-352. DOI: $10.1016 /$ j.powtec.2020.07.053.

9. Dobromyslov A. V., Taluts N. I., Pilyugin V. P. Severe plastic deformation by high-pressure torsion of Hf and Hf-Ti alloys // International Journal of Refractory Metals and Hard Materials. 2020. - Vol. 93. - P. 105354. - DOI: 10.1016/j.ijrmhm.2020.105354.

10. Sun J., Ke Q., Chen W. Material instability under localized severe plastic deformation during high speed turning of titanium alloy Ti-6.5AL-2Zr-1Mo-1V // Journal of Materials Processing Technology. 2019. - Vol. 264. - P. 119-128. - DOI: 10.1016/j.jmatprotec.2018.09.002.

11. Bridgman P. W. Effects of High Shearing Stress Combined with High Hydrostatic Pressure // Physical Review. - 1935. - Vol. 48. - P. 825-847. - DOI: 10.1103/PhysRev.48.825.

12. Zhilyaev A. P., Langdon T. G. Using high-pressure torsion for metal processing: Fundamentals and applications // Progress in Materials Science. - 2008. - Vol. 53. - P. 893- 979. DOI: $10.1016 /$ j.pmatsci.2008.03.002.

13. Основы пластической деформации наноструктурных материалов / А. М. Глезер, Э. В. Козлов, Н. А. Конева, Н. А. Попова, И. А. Курзина / под ред. А. М. Глезера. М. : Физматлит, 2016. -304 с.

14. Non-equilibrium solid solution and nanocrystal structure of $\mathrm{Fe}-\mathrm{Cu}$ alloy after plastic deformation under pressure / V. A. Teplov, V. P. Pilugin, V. S. Gaviko, E. G. Chernyshov // Philosophical Magazine B. - 1993. - Vol. 68. - P. 877-881. - DOI: 10.1080/13642819308217944.

15. Nomura R., Uesugi K. Note: High-pressure in situ X-ray laminography using diamond anvil cell // Review of Scientific Instruments. - 2016. - Vol. 87. - P. 046105. - DOI: 10.1063/1.4948315.

16. Towle L. C., Riecker R. E. Shear Strength of Grossly Deformed Solids // Science. - 1969. Vol. 163. - P. 41-47. - DOI: 10.1126/science.163.3862.41.

17. In situ observation of the "crystalline $\Rightarrow$ amorphous state" phase transformation in $\mathrm{Ti} 2 \mathrm{NiCu}$ upon high-pressure torsion / R. V. Sundeev, A. V. Shalimova, A. M. Glezer, E. A. Pechina, M. V. Gorshenkov, G. I. Nosova // Materials Science and Engineering: A. - 2017. - Vol. 679. P. 1-6. - DOI: 10.1016/j.msea.2016.10.028.

18. Effect of high-pressure torsion on the structure and properties of the natural layered amorphous-crystalline Ti2NiCu composite / R. V. Sundeev, A. V. Shalimova, N. N. Sitnikov, O. P. Chernogorova, A. M. Glezer, M. Y. Presnyakov, I. A. Karateev, E. A. Pechina, A. V. Shelyakov // Journal of Alloys and Compounds. - 2020. - Vol. 845. - P. 156273. DOI: $10.1016 /$ j.jallcom.2020.156273. 
19. The formation, structure, and properties of the Au-Co alloys produced by severe plastic deformation under pressure / T. P. Tolmachev, V. P. Pilyugin, A. I. Ancharov, E. G. Chernyshov, A. M. Patselov // The Physics of Metals and Metallography. - 2016. - Vol. 117. - P. 135-142. DOI: $10.1134 /$ S0031918X16020125.

20. Shear stress in high-pressure torsion and vickers hardness of Au-Co alloys / T. P. Tolmachev, V. P. Pilyugin, A. M. Patselov, A. V. Plotnikov, R. V. Churbaev // AIP Conference Proceedings. 2020. - Vol. 2315. - P. 040046. - DOI: 10.1063/5.0036671.

21. Structural features of the Au-Co alloy after mechanical alloying at cryo- and room temperatures according to X-ray diffractometry / T. P. Tolmachev, V. P. Pilyugin, A. M. Patselov, Yu. V. Solov'eva, R. V. Churbaev, A. V. Plotnikov // Russian Physics Journal. - 2021. - (In press). 